\title{
Gastrointestinal disorders in COVID-19 patients: a great imitator
}

pISSN: 0853-1773 • elSSN: 2252-8083 https://doi.org/10.13181/mji.bc.204960 Med J Indones. 2021;30:166-9

Received: August 18, 2020

Accepted: December 03, 2020

Published online: January 20, 2021

Author's affiliations:

Department of Internal Medicine, Faculty of Medicine, Universitas Indonesia, Cipto Mangunkusumo Hospital, Jakarta, Indonesia

\section{Corresponding author:}

Ari Fahrial Syam

Division of Gastroenterology,

Department of Internal Medicine, Faculty

of Medicine, Universitas Indonesia,

Cipto Mangunkusumo Hospital, Jalan

Diponegoro No. 71, Kenari, Senen,

Central Jakarta 10430, DKI Jakarta,

Indonesia

Tel/Fax: +62-21-31930956

E-mail: ari_syam@hotmail.com

\begin{abstract}
Up to this point, the coronavirus disease 2019 (COVID-19) pandemic is still ongoing. Some studies with a large number of cases have reported its clinical manifestations, concluding that the disease is a great imitator. Patients may present with symptoms other than the main symptoms of respiratory tract infections, such as diarrhea, abdominal pain, nausea, or vomiting, which may sometimes cause a delayed treatment in managing COVID-19 patients. Reports of various hospitals have also demonstrated gastrointestinal complaints as a clinical manifestation in those patients. The patients may come with gastrointestinal symptoms as their early clinical manifestation, or the gastrointestinal symptoms may be found in hospitalized COVID-19 patients, which indeed can be explained since the SARS-CoV-2, an etiologic agent of COVID-19 infection, can obviously be found along the gastrointestinal tract. Hence, the virus can be found in fecal and anal, and therefore, rectal swabs can be used as a diagnostic tool for COVID-19 infection.
\end{abstract}

KEYWORDS COVID-19, gastrointestinal disease, SARS-CoV-2
Coronavirus disease 2019 (COVID-19) infection has become a global problem, and the World Health Organization declared it as public health emergency of international concern on January 31, 2020. Until the end of October 2020, more than 40 million people have been infected, with 1,150,000 deaths worldwide. As we have known, the COVID-19 infection is caused by a new pathogen, which has been immediately identified as a novel family of the Betacoronavirus genus. It is highly associated with some coronavirus found in bats and with severe acute respiratory syndrome coronavirus (SARS-CoV), which is known as SARS-CoV-2. ${ }^{1}$ Further biomolecular research on the structure of the virus has found that the main target for developing vaccine, treatment, and diagnostic tools is the glycoprotein spike of CoV. ${ }^{2}$

During the initial spread of COVID-19 infection, the early manifestation was lung infection, i.e., pneumonia.
The main symptoms of COVID-19-caused pneumonia are fever, cough, and dyspnea; in addition, mild-tomoderate complaints are also noted, such as headache, nasal congestion, sore throat, myalgia, and arthralgia. ${ }^{3,4}$ A study conducted by $\mathrm{Xu}$ et $\mathrm{al}^{5}$ found that fever only occurred in $77 \%$ cases, while cough was found in $81 \%$ cases; diarrhea, $8 \%$; and dyspnea, $3 \%$. The cases actually involved mild symptoms during treatment within 1-4 days after their onset. Moreover, the symptoms developed after the exposure to the disease within the range of 3-5 days. ${ }^{5}$

On the next natural history of the disease, the COVID-19 patients were not diagnosed as having COVID-19 infection at first, since their first symptoms were gastrointestinal, presenting with stomachache and diarrhea, which tends to lead the diagnosis to gastrointestinal infection. When we look further into the pathophysiology of the disease, we find that the 
virus can actually involve various organs that contain angiotensin-converting enzyme 2 (ACE2) receptors. ${ }^{6}$ The virus will penetrate into the organs through those receptors. ACE2 receptors have been identified as important regulators in intestinal inflammation. So, the COVID-19 infection in intestinal tissues may lead to a gastrointestinal disorder. However, it should be noted that the number of ACE2 receptors is actually lesser in the lung than in other body tissues; nevertheless, the main spread of the virus is through droplet transmission, indicating a co-receptor factor or other factors, which are expressed by the host. ${ }^{7}$

\section{Gastrointestinal symptoms}

Reports from China have demonstrated that onethird of the cases have involved diarrhea complaints. Patients may present with fever and diarrhea, while the cough and dyspnea symptoms may follow later. ${ }^{8}$

In the case of a 23-year-old young patient who had been confirmed positive with COVID-19 infection, from Indonesia, the gastrointestinal symptoms have been found to occur concomitantly with upper respiratory tract symptoms. The patient has also experienced epigastric pain in addition to fever, cough, and dyspnea as well as nausea and vomiting. ${ }^{3}$ A study from China has also reported a young, 22-year-old patient who was admitted to hospital, with fever and diarrhea 4 days before the admission. Like the patient from Indonesia, this patient has also been trying to take some medicine for diarrhea first before seeking treatment in the hospital. No positive result was found during stool evaluation; however, the chest $\mathrm{X}$-ray revealed infiltrates on both lungs. The patient also had a travel history to Wuhan, which is the epicenter of COVID-19 virus transmission in China. The nasopharyngeal swab confirmed the presence of the SARS-CoV-2 virus. ${ }^{4}$

In patients with gastrointestinal symptoms, such as diarrhea, the virus is also found in fecal specimens. However, the fecal-oral transmission of the virus, which is similar to the transmission of typhoid fever or rotavirus infection in the gastrointestinal tract, has not been confirmed so far. However, it is interesting to note that in patients with early symptoms of diarrhea, the virus tends to persist inside the body longer than those without gastrointestinal symptoms. A study conducted by Jin et $\mathrm{al}^{6}$ investigated 74 patients with COVID-19 infection and gastrointestinal symptoms, such as diarrhea, nausea, and vomiting. Interestingly, the study showed that actually $28 \%$ of the patients who had gastrointestinal symptoms had no respiratory ones. When they were compared to a group of patients without gastrointestinal symptoms, which were 577 patients, patients with gastrointestinal symptoms have been found to have more severe complications, a fever higher than $38.5^{\circ} \mathrm{C}$, a greater number of family members who got infected, and a greater number of patients with liver dysfunction, which was characterized by an increase of alkaline phosphatase or aspartate aminotransferase levels. ${ }^{4}$

In general, the existing reports have demonstrated that gastrointestinal symptoms in COVID-19 patients had varied incidence rates. The symptoms of diarrhea can be found generally in $2-10 \%$ of cases, whereas nausea occurs in $2-15 \%$, vomiting in $1-5 \%$, and abdominal pain in $2-6 \%$. Overall, most cases, up to $80 \%$, have loss of appetite. Moreover, COVID-19 patients usually have a loss of smell and taste, exacerbating their loss of appetite.

Another study from China has also evaluated cases with gastrointestinal complaints, finding that of the 95 studied cases, 58 (61.6\%) had gastrointestinal symptoms, which included diarrhea in $24.2 \%$ cases, nausea in $17.9 \%$, vomiting in $4.2 \%$, and liver dysfunction in $32.6 \%$. Another study in China with 140 patients showed that fever was found in $91.7 \%$ of cases, while cough was found in $75 \%$ of cases and other gastrointestinal symptoms in $40 \%$, and it also suggested that diabetes mellitus and hypertension were the most common comorbidities. ${ }^{9}$

\section{COVID-19 and inflammatory bowel disease}

There is an interesting issue on the correlation between COVID-19 infection and inflammatory bowel disease (IBD). A hypothesis has assumed that a patient who is experiencing the active phase of IBD is at risk of having COVID-19 infection as the inflammation will increase the ACE2 expression. Results of studies with a small number of samples of patients with Crohn's disease have demonstrated a little increase in DPP4 and TMPRSS2 gene expression and reduced expression of ANPEP compared to the control. Therefore, we can speculate that IBD-associated inflammation itself does not increase the expression of factors that may allow the extrapulmonary SARS-CoV-2 transmission. ${ }^{10}$

In Northern Carolina from March 4, 2020 to April 14, 2020, 14,235 individuals were tested for SARSCoV-2, $8.2 \%$ of whom tested positive. Among them, the prevalence of IBD was $1.2 \%(168 / 14,235)$, and among 
these 168 IBD patients, the prevalence of COVID-19 was $3.0 \%$ (5/168). From this study using multivariate logistic regression analysis, only those aged $>66$ years was found to be independently associated with an increase risk of COVID-19. ${ }^{11}$

\section{The existence of the virus in the gastrointestinal tract}

As we have known, COVID-19 infection is caused by a virus named SARS-CoV-2. Similar to the SARS-CoV and MERS-CoV, this novel coronavirus is classified into the Betacoronavirus group. ${ }^{12}$ Up to this point, researchers have identified that other than through the respiratory tract, nasopharyngeal swab, bronchoalveolar lavage, nasal swab, sputum, and blood, the virus can also be collected from fecal specimens.

In a study of six patients with gastrointestinal issues, endoscopic evaluation and biopsy were performed, and the virus was found in several gastrointestinal sites, including the esophagus, stomach, duodenum, and rectum. Among them, assessment for virus from fecal specimens had also been performed, finding that $52.4 \%$ of them had positive results of virus on their fecal specimens. ${ }^{13}$ Thus, it provides another confirmation that the virus can attach to the gastrointestinal tract and can be found in fecal specimens. In a study in Singapore, the virus was found in fecal specimens for $50 \%$ of cases; nevertheless, in COVID-19 patients with positive results in their fecal specimens, the gastrointestinal symptoms only manifested in half of the cases (Table 1). ${ }^{14}$ In a study evaluating the distribution of virus in various samples of the body, half of COVID-19-associated pneumonia patients who are also positive for COVID-19 also had the virus in their fecal specimens. ${ }^{15}$ The virus can be detected in $32.8 \%$ of COVID-19 cases, which indicates that the SARS-CoV-2 virus can survive in human intestines and fecal route transmission may occur. ${ }^{16}$ In contrast, Lo et al ${ }^{17}$ detected

Table 1. The positivity rate of RNA virus SARS-CoV-2 in fecal specimens

\begin{tabular}{lccc}
\hline $\begin{array}{l}\text { First author, } \\
\text { year }\end{array}$ & Design & $\begin{array}{c}\text { Positive } \\
\text { test result } \\
\text { (total test) }\end{array}$ & $\begin{array}{c}\text { Positivity } \\
\text { rate (\%) }\end{array}$ \\
\hline Young, $^{14} 2020$ & Cross-sectional & $4(8)$ & 50 \\
\hline Wang, $^{15} 2020$ & Cross-sectional & $44(153)$ & 29 \\
Lo, $^{17} 2020$ & Retrospective study & $9(10)$ & 90 \\
Xie $^{18} 2020$ & Cross-sectional & $8(9)$ & 88.89 \\
\hline Chen ${ }^{19} 2020$ & Retrospective study & $28(42)$ & 66.67 \\
\hline
\end{tabular}

90\% of virus RNA COVID-19 from fecal samples using qualitative real-time reverse transcriptase-polymerase chain reaction assay.

In another study from China, a total of 19 suspected cases' stool samples were collected at Sichuan Provincial People's Hospital (10 patients) and Sichuan Mianyang 404 Hospital (9 patients): among 9 confirmed patients, 8 stool samples showed positive results for 2019-nCoV; interestingly, the virus could still be detected in stool samples from patients without diarrhea symptoms. ${ }^{18}$

In addition to the findings in fecal specimens, the SARS-CoV-2 virus can also be identified using anal or rectal swab. Upon further evaluation, the rectal swab actually ranks second in positive detection rate. Therefore, rectal swab can be utilized in COVID-19 cases that have gastrointestinal manifestation. But more studies are urgently needed to better characterize the magnitude of SARS-CoV-2 viral persistence in feces and its potential for disease transmission and possible implications for COVID-19 discharge and isolation policies in the future.

In conclusion, gastrointestinal disorder has become the clinical manifestation of concern in COVID-19 infection, both as an early and as an additional symptom. From a pathophysiological point of view, the SARS-CoV-2 virus can obviously be found along the gastrointestinal tract, which is supported by the fact that the virus can be detected in fecal specimens. Moreover, anal or rectal swab may serve as a diagnostic tool for COVID-19 cases that have gastrointestinal symptoms.

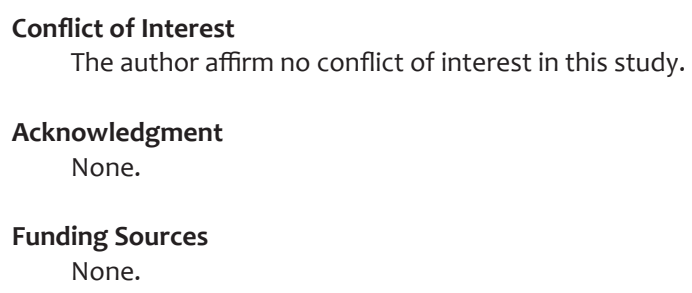

\section{REFERENCES}

1. Wu F, Zhao S, Yu B, Chen YM, Wang W, Song ZG, et al. A new coronavirus associated with human respiratory disease in China. Nature. 2020;579:265-9.

2. Wrapp D, Wang N, Corbett KS, Goldsmith JA, Hsieh CL, Abiona O, et al. Cryo-EM structure of the 2019-nCoV spike in the prefusion conformation. Science. 2020;367(6483):1260-3.

3. Azwar MK, Kirana F, Kurniawan A, Handayani S, Setiati S. Gastrointestinal presentation in Covid-19 in Indonesia: a case report. Acta Med Indones. 2020;52(1):63-7.

4. Song $Y$, Liu P, Shi XL, Chu YL, Zhang J, Xia J, et al. SARS-CoV-2

mji.ui.ac.id 
induced diarrhoea as onset symptom in patient with COVID-19. Gut. 2020;69(6):1143-4.

5. Xu XW, Wu XX, Jiang XG, Xu KJ, Ying $L J, M a C L$, et al. Clinical findings in a group of patients infected with the 2019 novel coronavirus (SARS-Cov-2) outside of Wuhan, China: retrospective case series. BMJ. 2020;368:m606.

6. Jin X, Lian JS, Hu JH, Gao J, Zheng L, Zhang YM, et al. Epidemiological, clinical and virological characteristics of 74 cases of coronavirus-infected disease 2019 (COVID-19) with gastrointestinal symptoms. Gut. 2020;69(6):1002-9.

7. Qi F, Qian S, Zhang S, Zhang Z. Single cell RNA sequencing of 13 human tissues identify cell types and receptors of human coronaviruses. Biochem Biophys Res Commun. 2020;526(1):135-40.

8. Xiao F, Tang M, Zheng X, Liu $\mathrm{Y}$, Li X, Shan H. Evidence for gastrointestinal infection of SARS-CoV-2. Gastroenterology. 2020;158(6):1831-3.e3.

9. Zhang JJ, Dong X, Cao YY, Yuan YD, Yang YB, Yan YQ, et al. Clinical characteristics of 140 patients infected with SARS-CoV-2 in Wuhan, China. Allergy. 2020;75(7):1730-41.

10. Monteleone G, Franzè E, Laudisi F. Expression of receptors for SARS-CoV-2 in the gut of proteins with inflammatory bowel disease. Gut Liver. 2020;14(4):530-1.

11. Gubatan J, Levitte S, Balabanis T, Patel A, Sharma A, Habtezion A. SARS-CoV-2 testing, prevalence, and predictors of COVID-19 in patients with inflammatory bowel disease in Northern
California. Gastroenterology. 2020;159(3):1141-4.e2

12. Abebe EC, Dejenie TA, Shiferaw MY, Malik T. The newly emerged COVID-19 disease: a systemic review. Virol J. 2020;17(1):96.

13. Lin L, Jiang X, Zhang Z, Huang S, Zhang Z, Fang Z, et al. Gastrointestinal symptoms of 95 cases with SARS-CoV-2 infection. Gut. 2020;69(6):997-1001.

14. Young BE, Ong SWX, Kalimuddin S, Low JG, Tan SY, Loh J, et al. Epidemiologic features and clinical course of patients infected with SARS-CoV-2 in Singapore. JAMA. 2020;323(15):1488-94.

15. Wang $\mathrm{W}, \mathrm{Xu} \mathrm{Y}$, Gao R, Lu R, Han K, Wu G, et al. Detection of SARS-CoV-2 in different types of clinical specimens. JAMA. 2020;323(18):1843-4.

16. Bwire GM, Majigo MV, Njiro BJ, Mawazo A. Detection profile of SARS-CoV-2 using RT-PCR in different types of clinical specimens: a systematic review and meta-analysis. J Med Virol. 2020;10.1002/jmv.26349.

17. Lo IL, Lio CF, Cheong HH, Lei Cl, Cheong TH, Zhong X, et al. Evaluation of SARS-CoV-2 RNA shedding in clinical specimens and clinical characteristics of 10 patients with COVID-19 in Macau. Int J Biol Sci. 2020;16(10):1698-707.

18. Xie C, Jiang L, Huang $\mathrm{G}$, Pu H, Gong B, Lin H, et al. Comparison of different samples for 2019 novel coronavirus detection by nucleic acid amplification tests. Int J Infect Dis. 2020;93:264-7.

19. Chen Y, Chen L, Deng Q, Zhang G, Wu K, Ni L, et al. The presence of SARS-CoV-2 RNA in the feces of COVID-19 patients. J Med Virol. 2020;92(7):833-40. 